\title{
RISKGATE, una herramienta diseñada para la minería de carbón en la mejora de la seguridad, eficiencia y a nivel operacional
}

\author{
RISKGATE a tool designed for coal mining in improving safety, efficiency and \\ operational level
}

\author{
Philipp Kirsch \\ Centro de seguridad y salud en la industria minera de la Universidad de Queensland. Brisbane. Australia. \\ Recibido: 26-03-14 \\ Aceptado: 05-05-14 \\ Correspondencia: \\ Philipp Kirsch \\ Centro de seguridad y salud en la industria minera de la Universidad de Queensland. \\ Brisbane. Australia \\ Correo electrónico: p.kirsch@uq.edu.au
}

Resumen

RISKGATE es un proyecto que brinda una herramienta interactiva en línea (página web) en la que mineras de carbón en Australia han hecho parte y construido un cuerpo de conocimiento a través de talleres de acción en investigación conformados por expertos industriales en tópicos específicos en áreas involucradas en seguridad y salud ocupacional a los que pueden estar expuestos los trabajadores como principal objetivo, sin embargo también incluye los peligros que pueden afectar la producción y maquinaria. RISKGATE cubre tópicos en el manejo de riesgos en las siguientes áreas: neumáticos, colisiones, incendios, procesos de aislamiento para el control de energía, control de estratos de suelo en las minas subterráneas, control de suelo en las minas de cielo abierto, explosiones, explosivos usados en minas subterráneas, explosivos aplicados en minas de cielo abierto, trabajos manuales, resbalones/tropiezos/caídas, escapes de gas, fallas en los bloques de carbón y erupciones, interface humano-maquina, almacenamiento de escoria, higiene ocupacional, escapes de agua y como último tópico que va a ser lanzado en el año 2014 será salud ocupacional.

Med Segur Trab (Internet) 2014; 60 (235) 286-289

Palabras clave: Manejo de riesgos, trabajo seguro, minas de carbón.

\begin{abstract}
RISKGATE is a project that provides an interactive online tool (website) in which coal miners in Australia have become part and built a body of knowledge through workshops, action research comprised industry experts on specific topics in areas involved occupational safety and health which workers may be affected by exposure, It also includes hazards that can affect production and machinery. RISKGATE covers topics in risk management in the following areas: tires, collisions, fires, isolation procedures for energy control, strata control underground, ground control in open cut, explosions, explosives underground, explosives open cut, manual tasks, slips/trips/ falls, outburst, coal bumps \& bursts, human-machine interface, tailings dams, occupational hygiene, inrush. This year we are working on Fitness for work.
\end{abstract}

Key words: Risk management, work safety, coal mines

Med Segur Trab (Internet) 2014; 60 (235) 286-289 
El Instituto de Minerales Sostenibles de la Universidad de Queensland fue establecido en el 2001, su investigación cubre todas las facetas del ciclo de vida de la minería desde la faceta de exploración hasta su cierre. Este está conformado por seis centros de investigación cuyas disciplinas van enfocadas a las comunidades, ambiente, seguridad y producción. El instituto tiene aproximadamente 350 empleados incluyendo 100 estudiantes de postgrado. Para mayor información puede dirigirse a http: //www.smi.uq.edu.au

El centro de seguridad y salud en la industria de minera (MISHC) del instituto de minerales sostenible es un centro internacionalmente reconocido en el área de investigación en las áreas de riesgo, salud y seguridad en la educación de la industria minera a nivel global. Con un enfoque aplicado a la investigación, los investigadores de MISHC se centran en los sistemas principales de práctica y procedimientos para resolver los retos que existen en salud y seguridad en una de las industrias de mayor riesgo a nivel mundial. Para ampliar información puede dirigirse a la página http://www.mishc.uq.edu.au

En el 2010, RISKGATE nació como proyecto de investigación por medio del apoyo financiero de la asociación de carbón en Australia (ACARP). El plan del proyecto era que tuviera una cobertura nacional y significativa en la construcción de la base de datos de conocimiento acerca de los mayores riesgos a nivel de seguridad industrial y salud ocupacional que se pueden presentar en la minería carbonífera. Este gran proyecto ha sido la mayor iniciativa que se ha llevado a cabo en la industria minera entorno a la investigación en las áreas de salud ocupacional y seguridad industrial. Durante los últimos tres años RISKGATE ha colectado conocimiento en el manejo de riegos por medio de talleres de acción en el desarrollo de tópicos específicos que forman parte de la industria, por medio de expertos industriales en las siguientes áreas: neumáticos, colisiones a nivel de vehículo-vehículo y/o vehículo-persona; incendios, procesos de aislamiento para el control en la liberación de energía; estratos de suelo en minas subterráneas, control de suelo en minas de cielo abierto, explosiones, explosivos usada en minería subterránea, explosivos usados en minería de cielo abierto, trabajos manuales y tropiezos/resbalones y caídas (tópicos que fueron lanzados en diciembre del 2012), en el año 2013 se adicionaron otros tópicos importantes para ser incluidos y analizados, tales como escapes de gas, fallas en los bloques de carbón y erupciones, interface humano-maquina, almacenamiento de escoria, higiene ocupacional y escapes de agua. Para el presente año se está trabajando en el tópico de salud ocupacional siguiendo las misma metodología que han seguidos los anteriores tópicos.

El proyecto ha sido dirigido por Profesor Asociado Philipp Kirsch quien pertenece al grupo de MISHC, con el soporte de Dr. Jill Harris y Dr. David Cliff. Otros líderes y expertos en manejo de estos tópicos reflejados en la página web son Bruce Hebblewhite y Duncan Chalmers (pertenecientes de la universidad de New South Wales), David Williams, Robin Burgess-Limmerick y Gul Kizil (pertenecientes de la Universidad de Queensland), Tilman Rasche (del gobierno de Queensland) y Peter Bergin, Jim Galvin y Alastair Torrance (consultantes independientes). Muchas de las compañías de minería de carbón han sido también involucradas en el proyecto, como Anglo American Metallurgical Coal, Glencore/Xstrata, Peabody Energy, BMA/BHP, Centennial Coal, Adani, Bandanna Energy Resources, Caledon Resources, Rio Tinto y Gujarat NRE. Para este proyecto más de 100 expertos de diferentes compañías han invertido más de 400 días de su tiempo para trabajar en el proyecto RISKGATE.

El proyecto comenzó en el 2010 con dos talleres industriales para el desarrollo del plan de procedimiento. De este modo, la industria definió los resultados y la estructura del proyecto, lo que se presentó a la institución que se encargaría de soportar el proyecto financieramente, en este caso ACARP. En el 2011, el sistema en línea (página web) fue diseñado y revisado por medio de la contribución de la industria. Al mismo tiempo, la acción de estructura del ciclo del taller de investigación fue desarrollada alrededor de la metodología de "corbatín" (ver Figura 1) para la captura del conocimiento de los expertos industriales acerca de los nominados peligros que se iban a analizar. El sistema y el proceso de los talleres de acción investigativa analizan el evento de iniciativa como punto inicial en la investigación y al transcurrir la investigación se identifican las causas del 
porque sucedió el evento, después de analizar las causas se analizan los posibles controles preventivos con el fin de evitar o minimizar aquel evento no deseado. Al otro lado del corbatín se enfoca en las consecuencias del evento y los controles de mitigación que puedan controlar aquellas secuelas, o minimizar el grado de gravedad de las consecuencias que pueden afectar a personas, producción y maquinaria. Cada tópico tiene una continua revisión en cada ciclo de un nuevo taller que se realizan.

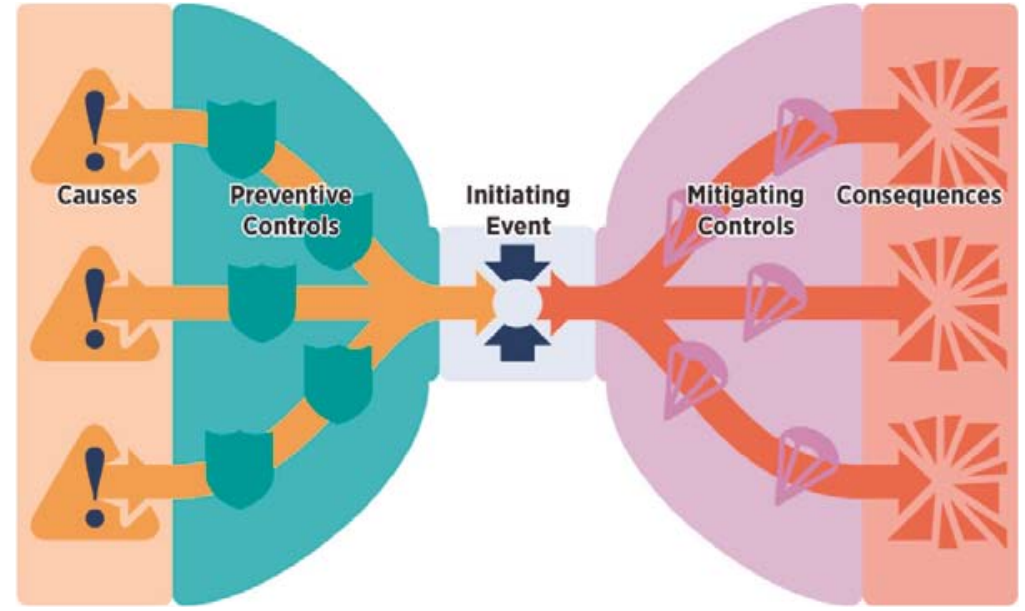

Figura 1. Análisis de modelo esquemático corbatín que fue usado como modelo para desarrollar RISKGATE

Este proyecto es valioso para cada individuo u organización que necesite del manejo de estos tipos de riesgos y por lo cual puedan alcanzar el manejo y control del riesgo a analizar. El conocimiento puede ser usado para asistir con la evaluación de riesgos, para los auditores en los procesos de operación, investigación de incidentes y sistemas de manejo de desarrollo y entrenamiento en los tópicos disponibles. El equipo de RISKGATE ha desarrollado videos, casos de estudio y en el uso de guías para asistir en la comprensión e implementación de la metodología de "corbatín" para alcanzar el manejo de riesgo en el sistema de la corporación y en las operaciones del sitio minero.

La página principal donde aparecen los tópicos de RISKGATE están disponibles con una descripción del tópico individualmente en la página web y cualquier persona puede verlo por medio de internet. Sin embargo, para tener acceso a información tal como las causas, controles preventivos, controles de mitigación y las consecuencias relacionadas a los eventos de iniciativa o «eventos no deseados» de cada tópico que es suministrada por el método interactivo "corbatín" está solamente se encuentra accesible para las compañías Australianas de carbón y a los investigadores. Esto no quiere decir que este sistema no se pueda usar en otro país. En este momento RISKGATE ha sido desarrollado por la industria de carbón en Australia para la industria de carbón de este país. Sin embargo, es posible la traducción del sistema en otro idioma y ser adaptada en otro país para otras mineras carboníferas, por lo tanto es crítico validar la información con expertos locales adaptando los métodos de minería de la nueva región a ser implementado. Por tal razón se requiere una evaluación inicial y adaptación con los expertos para ser adaptado ese conocimiento al sistema y a los métodos de minería que se llevan en el país. Algunos enfoques serán diferentes, por lo tanto es fundamental validar que la información sea correcta en el nuevo entorno. Para cualquier inquietud por favor contacte el equipo de RISKGATE para discutir el acceso al sistema.

Los futuros planes de RISKGATE se centran en que sea disponible y continuamente actualizado y mantenido en un futuro previsible. RISKGATE es uno de los mayores logros en el área de salud y seguridad que se haya desarrollado en la industria carbonífera en Australia. Este logro es aplicable en cualquier otra industria que desee unirse y compartir sus conocimientos acerca del manejo de riesgos, para que este conocimiento compartido 
llegue a encontrarse disponible a todos los miembros de las industrias, y los resultados de salud y seguridad puedan ser beneficiarios a nivel de industria.

||||||||||||||||||||||||||||||||||||||||||||||||||||||||||||||||||||||||||||||||||||||||||||||||||||||||||||||||||||||||||||||||||||||||||||||||||||||||||||||||||||||||||||||||||||||||||||||||||||||||||||||||||||||||||| 